\title{
Effect of Sex on Flavor-related and Functional Compounds in Freeze-dried Broth Made from Korean Native Chicken
}

\author{
Dinesh D. Jayasena ${ }^{1,2}$, Samooel Jung ${ }^{1}$, Hyun Joo Kim, Amali U. Alahakoon ${ }^{1}$, \\ Ki Chang $\mathrm{Nam}^{3}$, and Cheorun Jo* \\ Department of Agricultural Biotechnology, Center for Food and Bioconvergence, and \\ Research Institute of Agriculture and Life Sciences, Seoul National University, Seoul 151-921, Korea \\ ${ }^{1}$ Department of Animal Science and Biotechnology, Chungnam National University, Daejeon 305-764, Korea \\ ${ }^{2}$ Department of Animal Science, Uva Wellassa University, Badulla 90000, Sri Lanka \\ ${ }^{3}$ Department of Animal Science and Biotechnology, Sunchon National University, Suncheon 540-742, Korea
}

\begin{abstract}
Studies on the flavour characteristics of meat-based broth, quantification of flavour-related and functional compounds, and factors affecting the availability of such compounds are minimal. The present study was designed to determine the effects of sex on flavor-related and functional compounds in freeze-dried broth (FDB) made from Korean native chickens (KNC). Male and female KNC from a commercial strain (Woorimatdag ${ }^{\mathrm{TM}}$ ) were reared under similar commercial conditions. FDB was separately prepared using male and female birds aged $100 \mathrm{~d}$ (six birds of each sex) and analyzed for nucleotide, free amino acid, betaine, carnitine, carnosine, anserine, and creatine contents, and fatty acid composition. The levels of betaine, carnitine and creatine in FDB were not significantly different between the two sexes $(p>0.05)$ in KNC. Carnosine and anserine were not detected in FDB samples. However, FDB from female chickens had significantly higher inosine-5monophosphate and arachidonic acid contents than did FDB from male chickens. FDB prepared with male KNC contained higher levels of inosine, linoleic acid, glycine, alanine, lysine, and serine $(p<0.05)$. However, glutamic acid, oleic acid, and DHA were present in comparable amounts $(p>0.05)$ in FDB made from male and female KNC. Our findings suggest that the sex of KNC has significant effect on the contents of flavor-related compounds, but not functional compounds.
\end{abstract}

Keywords: betaine, carnitine, freeze-dried broth, inosine-5-monophosphate, Korean native chicken

\section{Introduction}

Food and its consumption patterns are coupled with the unique culture of Korean consumers. Meat consumption has increased consistently during the past few decades in Korea, and it is often consumed either roasted or as soups or stews (Nam et al., 2010). Chicken is a dominant type of meat in the Korean market, and it is typically served roasted or braised with vegetables or in soups. For instance, samgyetang and baeksuk, made from young and mature Korean native chickens (KNC), are popular traditional soups among Korean consumers (Nam et al., 2010).

Soup is generally prepared from meat, fish, or vegeta-

\footnotetext{
*Corresponding author: Cheorun Jo, Department of Agricultural Biotechnology, Center of Food and Bioconvergence, and Research Institute of Agriculture and Life Sciences, Seoul National University, Seoul 151-921, Korea. Tel.: +82-2-880-4804, Fax: +82-2-873-2271, E-mail: cheorun@snu.ac.kr
}

ble stock combined with various other ingredients (Gadekar et al., 2009). It can be consumed both as a food source and as a beverage. Soups are consumed primarily owing to their mild flavor and high satiety value (Chiang et al., 2007). The characteristic flavor of a soup is mainly attributed to non-volatile flavor components such as free amino acids, 5-nucleotides, and soluble sugars, as well as volatile compounds (Chiang et al., 2007). Soups are often served as folk remedies in many countries (Ke et al., 2011) and are excellent for patients recovering from illnesses, likely because they have high digestibility and nutritive value together with low-fat content (Gadekar et al., 2009). Furthermore, soups stimulate the secretion of saliva and therefore improve appetite. According to Ke et al. (2011), chicken soup is the most ubiquitous medicinal soup in the world. During the summer, samgyetang is popular among Korean consumers as it is believed to reduce body heat (Nam et al., 2010).

Carnitine, betaine, carnosine, anserine and creatine are 
well-known functional compounds present in meat. Carnitine ( $\beta$-hydroxy- $\gamma$-trimethyl amino butyric acid) is a key member of the fatty acid metabolic pathway in animals, transporting long-chain fatty acids across the inner mitochondrial membrane for $\beta$-oxidation. Carnitine also buffers excess acetyl groups that are produced in the mitochondria (Constantin-Teodosiu et al., 1996). Betaine acts as an osmolyte to protect cells, proteins, and enzymes from environmental stress. Furthermore, it possesses the ability to improve growth performance and fat distribution of livestock in addition to its crucial role as a methyl donor in the methionine cycle (de Zwart et al., 2003). Histidine-derived dipeptides such as carnosine ( $\beta$-alanylL-histidine) and anserine (N- $\beta$-alanyl-1-methyl-L-histidine) are the most abundant antioxidants in meat and well known for their potent buffering properties (Guiotto et al., 2005). Moreover, they possess a role in wound healing, recovery from fatigue and prevention of diseases related to stress (Young et al., 2013). More importantly, these vital dipeptides are only found in meat, poultry and some fish, but not in foods of plant origin (Young et al., 2013). The amino acid derivative, creatine, is synthesized from arginine, methionine and glycine and together with creatine phosphate it supplies the energy required for vigorous muscle contraction (Wyss and Kaddurah-Daouk, 2000). Furthermore, creatine can contribute certain sensory properties to food such as the overall flavor of meat extracts (Mora et al., 2010).

The flavor of chicken meat is affected by several factors, including breed/strain of the chicken, diet, presence of free amino acids and nucleotides, and the cooking technique used (Jayasena et al., 2013a). Many studies have shown that species, breed, sex, muscle type, and cooking technique govern the contents of aforementioned functional compounds (de Zwart et al., 2003; Wyss and Kaddurah-Daouk, 2000). Hence, the flavor of meat-based soups and the contents of flavor-related and functional compounds in these soups may be influenced by the same factors, due to the fact that the main raw ingredient is the meat. Wei et al. (2012) recently showed that soup prepared with an indigenous chicken breed had the best overall flavor with nucleotide content higher than that prepared with a commercial broiler breed. On-going research has also demonstrated that freeze-dried broth (FDB) from KNC contained significantly higher levels of flavor-related compounds such as inosine-5-monophosphate (IMP) and cysteine, and functional compounds such as betaine and carnitine, compared to commercial broilers. Chicken broth cubes have higher levels of glutamic acid than does pork broth cubes, but less IMP (Chiang et al., 2007). However, only a small portion of the nutrients migrate from meat into the soup during the boiling process (Ke et al., 2011).

Scientific studies on the flavor characteristics of meatbased soups, quantification of flavor-related and functional compounds, and factors affecting the availability of such compounds are minimal, and no studies regarding the effect of sex on the quantity of flavor-related and functional compounds in FDB are available. Therefore, the present study was designed to determine the effect(s) of sex on the contents of flavor-related and functional compounds in FDB made from KNC.

\section{Materials and Methods}

\section{Animals and rearing}

A total of $36 \mathrm{~d}$-old-chicks (18 chicks of each sex) from a certified meat-type commercial KNC strain (Woorimatdag) were allotted to three floor pens (six chicks of each sex per pen) within a single house at a commercial chicken farm (Gimcheon, Korea) with suitable husbandry conditions. During the experimental period, KNC were reared under similar standard commercial conditions. Additionally, the chicken care facilities and procedures were carried out to meet or exceed the standards established by the Committee for Accreditation of Laboratory Animal Care at National Institute of Animal Science in Korea. Chicks were fed commercial starter $[3,100 \mathrm{kcal} \mathrm{ME} / \mathrm{kg}$, $23 \%$ crude protein $(\mathrm{CP})$ during the first $7 \mathrm{~d}$ ], grower $\left(3,200 \mathrm{kcal} \mathrm{ME} / \mathrm{kg}, 20 \% \mathrm{CP}\right.$ from $8^{\text {th }}$ to $\left.21^{\text {st }} \mathrm{d}\right)$, and finisher $\left(3,200 \mathrm{kcal} \mathrm{ME} / \mathrm{kg}, 18 \% \mathrm{CP}\right.$ from $22^{\text {nd }} \mathrm{d}$ onwards) diets ad libitum. Furthermore, the birds had free access to water during the experiment period, but had no access to the outdoor environment. All experimental procedures conducted during this experiment followed the recommendations described in "The Guide for the Care and Use of Laboratory Animals" published by the Institutional Animal Care and Use Committee (IACUC) of the NIAS (2012-C-037) in Korea.

\section{Processing}

Two KNC of each sex were randomly selected from each of the three replicate pens (a total of six KNC from each sex) at $100 \mathrm{~d}$ of age. They were subjected to a $10 \mathrm{~h}$ feed withdrawal period and subsequently killed by a conventional neck cut. After bleeding for 2 min, the carcasses were defeathered and eviscerated manually, during which time the sex of each bird was confirmed. The car- 
casses were then chilled at $4^{\circ} \mathrm{C}$ for $24 \mathrm{~h}$, vacuum-packed and stored in a freezer at $20^{\circ} \mathrm{C}$ until further analysis. As the frozen storage period is short, it may not cause significant changes in the contents of flavor compounds.

\section{Preparation of freeze-dried broth}

The frozen carcasses of each sex were first thawed in a refrigerator $\left(4^{\circ} \mathrm{C}\right)$ for $24 \mathrm{~h}$ prior to the preparation of the FDB samples. The thawed carcasses were trimmed of the visible skin and fat, and boiled separately in water (1:1.5, $\mathrm{w} / \mathrm{v}$ ) for $40 \mathrm{~min}$. The cooked carcasses were then removed from the boiling water and the remaining broth samples were concentrated by further boiling for $2 \mathrm{~h}$. The concentrated broth samples were subsequently filtered through a testing sieve (wire diameter of $100 \mathrm{~mm}$, aperture of $150 \mathrm{~mm}$; Chunggye Sanggongsa, Korea) in order to remove the solid particles. After that, hexane was added $(1: 1, \mathrm{v} / \mathrm{v})$ to each filtrate and the mixtures were stirred using a magnetic stirrer (C-MAG HS 7, Ika Korea Co. Ltd., Korea) for $1 \mathrm{~h}$, and allowed to settle. The resulting supernatant layers (mainly fat) were carefully removed to separate containers and used for the analysis of the fatty acid composition. The remaining mixtures were finally lyophilized (TFD 5505, IL Shin Lab Co. Ltd., Korea) at $53^{\circ} \mathrm{C}$ under pressurized vacuum at 7 mTorr. The FDB samples were then stored in a freezer $\left(-80^{\circ} \mathrm{C}\right)$ until further analysis.

\section{Determination of carnosine, anserine, and creatine contents}

The contents of carnosine, anserine, and creatine were determined using the modified method of Jung et al. (2013). Each sample (2 g) was homogenized with $7.5 \mathrm{~mL}$ of $0.01 \mathrm{~N} \mathrm{HCl}$ (T25b, Ika Works, Malaysia) set at 13,500 rpm for $1 \mathrm{~min}$, and then centrifuged at 17,030 $\mathrm{g}$ for 15 min (HM-150IV, Hanil Co. Ltd., Korea). The supernatant $(250 \mathrm{~mL})$ was mixed with $750 \mathrm{~mL}$ acetonitrile. Following a storage period of $20 \mathrm{~min}$ at $4^{\circ} \mathrm{C}$ and centrifugation at $10,000 \mathrm{~g}$ for $10 \mathrm{~min}$, the supernatant was filtered through a $0.2 \mu \mathrm{m}$-polyvinylidene difluoride (PVDF) syringe filter (Whatman International Ltd., UK) and injected into an Atlantis HILIC silica HPLC column $(4.6 \times 150 \mathrm{~mm}, 3 \mu \mathrm{m}$; Waters Corp., USA) using a 1525 pump and a 717 plus autosampler (Waters Corp.). The partitioned fractions were detected using a 2487-diode array detector (Waters Corp.) at $214 \mathrm{~nm}$ to determine the creatine, carnosine, and anserine contents. A gradient elution was performed where mobile phase B was supplied at $1.2 \mathrm{~mL} \mathrm{~min}^{-1}$ for $16 \mathrm{~min}$ with a linear gradient (0 to $100 \%$ ) in a background of mobile phase A. Mobile phase A was $0.65 \mathrm{mM}$ ammonium acetate in water/acetonitrile $(25: 75, \mathrm{v} / \mathrm{v}, \mathrm{pH} 5.5)$, and phase B was $4.55 \mathrm{mM}$ ammonium acetate in water/ acetonitrile (70:30, v/v, pH 5.5). The content of each compound in a sample was determined using the standard curve derived from the respective standard (Sigma Corp., USA).

\section{Determination of betaine and carnitine contents}

Betaine and carnitine content in each FDB sample were measured using the modified method of Jayasena et al. (2014). The FDB samples (1.5 g) were first homogenized separately in $10 \mathrm{~mL}$ precipitating reagent (acetonitrile/methanol solution, 9:1, v/v) at 13,500 rpm for $30 \mathrm{~s}$ (Ika Works) and centrifuged at 2,090 $\mathrm{g}$ for $5 \mathrm{~min}$ (Hanil Co. Ltd.). The supernatant was then filtered into a $20-\mathrm{mL}$ volumetric flask through a funnel plugged with glass wool. This procedure was repeated after adding $10 \mathrm{~mL}$ precipitating reagent and the supernatant was again collected into the same volumetric flask. This filtrate was made up to $20 \mathrm{~mL}$ with the precipitating reagent and $2 \mathrm{~mL}$ of the resulting solution was thoroughly mixed with $0.810 \mathrm{~g}$ $\mathrm{Na}_{2} \mathrm{HPO}_{4}$ and $0.090 \mathrm{~g} \mathrm{Ag}_{2} \mathrm{O}(9: 1, \mathrm{w} / \mathrm{w})$ in a $15-\mathrm{mL}$ tube. The sample tubes were air-dried in a shaker without their caps for $30 \mathrm{~min}$, and then centrifuged at 2,090 $\mathrm{g}$ for 5 $\mathrm{min}$. The supernatant $(0.5 \mathrm{~mL})$ was added to $0.5 \mathrm{~mL}$ derivatizing reagent (1.39 $\mathrm{g}$ 2,4-dibromoacetophenone and $0.066 \mathrm{~g} 18$-crown-6 in $100 \mathrm{~mL}$ acetonitrile) in a $15-\mathrm{mL}$ tube and vortex-mixed. The mixture was heated at $80^{\circ} \mathrm{C}$ for $1 \mathrm{~h}$ in a water bath and then cooled for 5 min under running water. This mixture was filtered through a 0.2$\mathrm{mm}$ membrane filter and analyzed in an HPLC system to determine the betaine and carnitine contents. The HPLC system used was the same as that for determination of the carnosine, anserine, and creatine contents, except that the partitioned fractions were detected at $254 \mathrm{~nm}$. An isocratic elution was performed where the mobile phase was a 9:1 mixture of $25 \mathrm{mM}$ ammonium acetate, in which $\mathrm{pH}$ was adjusted to 3.0 using formic acid, and acetonitrile. The mobile phase was supplied at $1.4 \mathrm{~mL} \mathrm{~min}^{-1}$ for 20 min. Standards (betaine and L-carnitine hydrochloride) were obtained from Sigma Corp. The betaine and carnitine contents were calculated using a standard curve for each compound.

\section{Determination of nucleotide content}

Nucleotide content of each FDB sample was determined according to the method of Choe et al. (2010). The nucleic acids were extracted from the FDB samples $(0.5$ 
g each) using $25 \mathrm{~mL}$ of $0.7 \mathrm{M}$ perchloric acid. The $\mathrm{pH}$ of the extract was then adjusted to 7 with $5 \mathrm{~N} \mathrm{KOH}$. Subsequently, it was placed into a volumetric flask, and made up to a volume of $100 \mathrm{~mL}$ with $0.7 \mathrm{M}$ perchloric acid $(\mathrm{pH}$ 7.0). After $30 \mathrm{~min}$ of cooling, the mixture was centrifuged (Hanil Co. Ltd.) at $1,130 \mathrm{~g}\left(0^{\circ} \mathrm{C}\right)$, and the supernatant was filtered through a $0.2 \mu \mathrm{m}-\mathrm{PVDF}$ syringe filter (Whatman International Ltd.). The filtrate ( $5 \mathrm{~mL}$ ) was analyzed using a Waters-Atlantis dC18 reverse-phase HPLC column (4.6 $\times 250 \mathrm{~mm}, 5 \mu \mathrm{m}$ particle, Waters Corp.). The peaks of individual nucleotides were identified using the retention times for standards-hypoxanthine, inosine, inosine-5-monophosphate (IMP), adenosine-5-monophosphate (AMP; Sigma Corp.) and the concentrations were calculated using the area for each peak.

\section{Determination of fatty acid composition}

Total lipid content of the supernatant layer separated from each broth sample was extracted according to the method of Folch et al. (1957) with some modifications. Each sample was mixed $(1: 2, \mathrm{v} / \mathrm{v})$ with chloroform-methanol solution $(2: 1, \mathrm{v} / \mathrm{v})$ and stirred using a magnetic stirrer (Ika Korea Co. Ltd.) for $1 \mathrm{~h}$. Subsequently, each mixture was added with $40 \mathrm{~mL}$ of $0.88 \%(\mathrm{w} / \mathrm{v}) \mathrm{NaCl}$, stirred well and allowed to settle overnight. After removing the resulting supernatant layer containing methanol, the mixture was filtered through a No. 4 filter paper (Whatman International Ltd.). The remaining chloroform in the filtrate was evaporated by an Eyela N-1000 rotary evaporator (Tokyo Rikakikai Co. Ltd, Japan). The extracted oily solution was further evaporated by $\mathrm{N}_{2}$ gas $(99.999 \%)$ and used for the analysis of fatty acid composition.

After mixing the extracted oily samples $(0.5 \mathrm{~g})$ with 1 $\mathrm{N} \mathrm{KOH}$ in ethanol $(50 \mathrm{~mL})$, the mixture was heated in a water bath $\left(70^{\circ} \mathrm{C}\right)$ for $1 \mathrm{~h}$. After cooling, $10 \mathrm{~mL}$ of each sample was transferred to a $50-\mathrm{mL}$ tube, vortex-mixed with distilled water and hexane $(10 \mathrm{~mL}$ each), and centrifuged (Hanil Co. Ltd.) at 1,130 $\mathrm{g}$ for $3 \mathrm{~min}$. The supernatant was removed and the remaining mixture was then mixed with $6 \mathrm{~mL}$ of HCl-distilled water solution $(1: 1, \mathrm{v} /$ v) and $5 \mathrm{~mL}$ of hexane, vortex-mixed and centrifuged (Hanil Co. Ltd.) at 1,130 $\mathrm{g}$ for $3 \mathrm{~min}$. The resulting supernatant hexane layer was collected in a $15-\mathrm{mL}$ tube. A similar extraction was repeated after adding $5 \mathrm{~mL}$ of hexane to the remaining original mixture. The resulting supernatant hexane layer was again collected in to the same tube, which was then completely evaporated by $\mathrm{N}_{2}$ gas (99.99\%). Next, each tube was added with $1 \mathrm{~N} \mathrm{H}_{2} \mathrm{SO}_{4}$ in methanol $(5 \mathrm{~mL})$ and heated in a water bath $\left(50^{\circ} \mathrm{C}\right)$ for 1 h. After cooling, $2 \mathrm{~mL}$ of distilled water and $3 \mathrm{~mL}$ of hexane were added to the same tubes, which were then centrifuged (Hanil Co. Ltd.) at $1,130 \mathrm{~g}$ for $3 \mathrm{~min}$. The top hexane layer containing fatty acid methyl esters (FAME) was transferred to another $15-\mathrm{mL}$ tube, concentrated up to $1.5 \mathrm{~mL}$ using $\mathrm{N}_{2}$ gas $(99.999 \%)$, and dehydrated through anhydrous $\mathrm{Na}_{2} \mathrm{SO}_{4}$ in to a vial. Fatty acid composition was then analyzed using a gas chromatograph (HP 7890, Agilent Technologies, USA). A split inlet (split ratio, 100 :1) was used to inject the samples into a capillary column $(30 \mathrm{~m} \times 0.32 \mathrm{~mm} \times 0.25 \mathrm{~mm}$; Omegawax 320, Supelco, USA), and ramped oven temperature was used for the analysis $\left(150^{\circ} \mathrm{C}\right.$ for $5 \mathrm{~min}$, increased to $170^{\circ} \mathrm{C}$ at $5^{\circ} \mathrm{C} / \mathrm{min}$ and maintained for $8 \mathrm{~min}$, then increased to $190^{\circ} \mathrm{C}$ at $5^{\circ} \mathrm{C} /$ min and maintained for $15 \mathrm{~min}$ and finally increased to $220^{\circ} \mathrm{C}$ at $5^{\circ} \mathrm{C} / \mathrm{min}$ and maintained for $30 \mathrm{~min}$ ). The inlet temperature was $210^{\circ} \mathrm{C} . \mathrm{N}_{2}$ gas was used as the carrier gas at a constant flow rate of $0.7 \mathrm{~mL} / \mathrm{min}$.

\section{Determination of free amino acid content}

The FDB samples were analyzed for free amino acid content as described by Hughes et al. (2002) with some modifications. Each FDB sample (1.5 g) was mixed with $20 \mathrm{~mL}$ of $2 \%(\mathrm{w} / \mathrm{v})$ trichloroacetic acid solution and homogenized (Hanil Co. Ltd.) at 1,130 $\mathrm{g}$ for $30 \mathrm{sec}$. The homogenate was centrifuged (Ika Works) at $17,000 \mathrm{~g}$ for $30 \mathrm{~min}$ and filtered through a $0.45-\mu \mathrm{m}$ PVDF syringe filter (Whatman International Ltd.). The filtrate was derivatized using AccQ-Tag ${ }^{\mathrm{TM}}$ (Waters Corp.) according to the manufacturer's protocol, and $5 \mathrm{~mL}$ was injected into a reverse-phase HPLC column $(3.9 \times 150 \mathrm{~mm}$, Waters Corp.). The column temperature was $37^{\circ} \mathrm{C}$ and a Waters ${ }^{\mathrm{TM}} 2475$ fluorescent detector was used with $250 \mathrm{~nm}$ and $395 \mathrm{~nm}$ of excitation and emission wavelengths, respectively. The separation was done by using buffers: A (Waters AccQTag eluent) and B (60\%, v/v, acetonitrile). Individual amino acids were identified by comparison of their retention times with those of calibration standards (Sigma Corp.). Peak areas were processed using Millennium 32 software and the concentrations of individual amino acids were reported.

\section{Statistical analysis}

The content of flavor-related and functional compounds in the FDB made from male and female $\mathrm{KNC}$ is reported as mean and standard error from six independent FDB samples. The experimental data were subjected to an analysis of variance for a completely randomized design using the procedure of general linear model using SAS software 
system (Release 9.3, SAS Institute Inc., Cary, NC, USA). Comparisons of means were performed by Tukey's multiple range tests $(p<0.05)$.

\section{Results and Discussion}

Studies on the effect(s) of sex on the content of flavorrelated and functional compounds in meat-based broth are rare. Results from the few available studies were considered in the following sections to add context to our current findings.

\section{Carnosine, anserine and creatine contents}

During recent studies comparing male and female KNC, Jung et al. (2013) found comparable $(p>0.05)$ anserine and carnosine contents in meat, respectively. It has also been shown that sex had no significant effect on the carnosine content of bovine meat (Mateescu et al., 2012). In the present study, it was expected to observe the availability of these histidine-derived dipeptides in the FDB samples primarily due to their high water solubility (Jayasena et al., 2014; Purchas et al., 2004). A clear-cut depletion of these dipeptides with the cooking liquor during heat treatment of meat, particularly during boiling in water, has been reported in KNC meat (Jayasena et al., 2014), lamb and beef (Purchas et al., 2004). However, both carnosine and anserine were not detected in the FDB samples in this study, irrespective of the sex of KNC (Table 1). Wu and Shiau (2002) reported similar results in which freshwater clam and hard clam essences contained no carnosine and anserine contents. Further, eel essence had only very little carnosine with no anserine despite eel meat was rich in carnosine. In addition, Sri Kantha et al. (2000) detected minute amounts of carnosine in Conger eel extract and lobster extracts. In contrast, several other authors reported comparative contents of these dipeptides in meat-based essences and extracts. For instance, six chicken essences varied in carnosine and anserine contents from 0.08 to $1.62 \mathrm{~g} / \mathrm{kg}$ and from 0.36 to $4.37 \mathrm{~g} / \mathrm{kg}$, respectively (Wu and Shiau, 2002). In addition, the carnosine content of a commercially available chicken muscle extract ranged from 0.7 to $0.9 \mathrm{~g} / \mathrm{kg}$ whereas those of beef muscle extract and beef bone extract varied from 37.5 to $38.2 \mathrm{~g} / \mathrm{kg}$ and 0.2 to $1.1 \mathrm{~g} / \mathrm{kg}$, respectively (Sri Kantha et al., 2000). Therefore, the cooking conditions used in this study (boiling of meat for $40 \mathrm{~min}$ ) might not be sufficient to extract these dipeptides into the cooking liquor. Hence, further investigations are essential to determine the cause for the absence of carnosine and anserine in the FDB made with KNC meat.

The creatine content of the FDB prepared from male and female $\mathrm{KNC}$ are shown in Table 1. Males generally contain a higher proportion of fast-twitch Type IIB muscle fibers (Derave et al., 2010). Type II muscle fibers (glycolytic muscles) demand greater contents of phosphocreatine for immediate regeneration of ATP as they highly rely on anaerobic metabolism compared to Type I muscle fibers (oxidative muscles). This ultimately yields higher creatine contents in Type IIB muscle fibers (Mora et al., 2010; Wyss and Kaddurah-Daouk, 2000). As a result, a higher creatine content was expected during the present experiment in the meat of male $\mathrm{KNC}$ and thereby in the FDB made with male KNC than those of females. Nevertheless, sex was found to have no significant effect on the creatine content of the FDB made with the $\mathrm{KNC}$ in this study. Similar to the findings of the present study, five $\mathrm{KNC}$ strains showed comparable $(p>0.05)$ creatine contents in their meat between the sexes (Jung et al., 2013). The creatine content of beef broth was reported to vary between 6112 and $6600 \mathrm{mmol} / \mathrm{L}$ (Cambero et al., 2000).

\section{Carnitine and betaine contents}

The effect of sex on betaine and carnitine contents of FDB prepared with KNC is shown in Table 1. No significant difference was observed in the carnitine content between FDB samples prepared with male and female $\mathrm{KNC}(p>0.05)$. Here, a depletion may have occurred from $\mathrm{KNC}$ meat during preparation of the broth samples owing to the high water solubility of carnitine and prolonged boiling period (Jayasena et al., 2014), which resulted in a considerable amount of carnitine in both FDB samples in

Table 1. Functional compound contents $(\mathrm{g} / \mathrm{kg})$ of freeze-dried broth made from male and female Korean native chickens

\begin{tabular}{cccccc}
\hline \hline Sex & Carnitine & Betaine & Carnosine & Anserine & Creatine \\
\hline Male & 0.20 & 0.34 & -1 & - & 0.89 \\
Female & 0.21 & 0.36 & - & - & 1.09 \\
SEM $^{2)}$ & 0.02 & 0.03 & - & - & 0.07 \\
\hline
\end{tabular}

\footnotetext{
${ }^{1)}$ Not detected.

${ }^{2)}$ Standard error of the mean $(\mathrm{n}=12)$.
} 
the present study. Male $\mathrm{KNC}$ aged $14 \mathrm{wk}$ had an average carnitine content of $0.096 \mathrm{~g} / \mathrm{kg}$ in their meat (Jayasena et al., 2014). In addition, Schmid (2009) reported the carnitine content of chicken breast, beef steak, minced beef, and turkey meat as $0.10,0.65,0.88$, and $0.21 \mathrm{~g} / \mathrm{kg}$, respectively.

On-going research showed a clear-cut effect of sex on betaine content of KNC meat, in which female chickens had higher levels of betaine than did male chickens $(p<0.05)$, but no such difference $(p>0.05)$ was observed between FDB samples prepared with male or female KNC in the present study (Table 1). Further investigations are required to determine the cause of this discrepancy. Few studies have quantified the amount of betaine in meatbased broth. Chicken noodle soup and New England clam chowder soup contained approximately one-third and one-half the betaine content reported in the present study, respectively (Zeisel et al., 2003). Similar to carnitine, the considerable amount of betaine found in FDB samples irrespective of sex could be attributable to the high water solubility of betaine (de Zwart et al., 2003) and prolonged boiling period during preparation of the broth samples (Jayasena et al., 2014), causing significant loss of this compound from raw KNC meat during cooking.

\section{Nucleotide content}

Concentrations of ATP degradation products in FDB prepared from male and female $\mathrm{KNC}$ are shown in Table 2. Sex of the birds significantly affected the levels of all ATP degradation products in FDB prepared with KNC, except that of hypoxanthine (Table 2). In this regard, female chickens showed higher levels of AMP and IMP in FDB samples than did male chickens $(p<0.05)$. Similar results were observed by Jung et al. (2013); female KNC had significantly higher IMP levels in both breast and leg meat than did male KNC. IMP is one of the major flavor precursors of meat (Jayasena et al., 2013b). Post-mortem processes such as aging and cooking degrade IMP to inosine, which is further broken down to hypoxanthine and ribose. IMP plays an important role in the umami flavor in food (Jayasena et al., 2013b), and thus the higher IMP content reported in FDB prepared with female KNC may result in better flavor characteristics. In the present study, male KNC had a significantly greater inosine content in FDB than did female KNC (Table 2). Cambero et al. (2000) quantified the contents of AMP, IMP, inosine, and hypoxanthine in beef broth at $95^{\circ} \mathrm{C}$ as $156,1081,551$, and $562 \mu \mathrm{mol} / \mathrm{L}$, respectively. Furthermore, Chiang et al. (2007) found that chicken broth cubes had significantly lower IMP content $(1.11 \mathrm{~g} / \mathrm{kg})$ than did pork broth cubes $(1.33 \mathrm{~g} / \mathrm{kg})$, both of which are much lower values than those reported in the present study for FDB (Table 2).

\section{Fatty acid composition}

The fatty acid composition of FDB prepared with male and female $\mathrm{KNC}$ is shown in Table 3 . The composition of most fatty acids in the FDB samples differed significantly between the sexes. No relevant studies regarding fatty acid compositions of meat-based broth were found to be comparative to the data reported in the present study. Oleic (C18:1), linoleic (C18:2), and palmitic (C16:0) acids were the predominant fatty acids in FDB, irrespective of sex (Table 3). Similarly, these fatty acids have been reported to be the main fatty acids in KNC meat (Choe et al., 2010). Oleic acid, the principal fatty acid detected in the FDB samples, is one of the major fatty acids associated with meat flavor (Choe et al., 2010). However, the oleic acid composition in FDB samples was comparable between sexes $(p>0.05)$. FDB prepared with male KNC showed significantly higher levels of myristic (C14:0) and palmitoleic (C16:1) acids than did FDB prepared with female KNC. However, the highest levels of palmitic ( $\mathrm{C}$ 16:0) and stearic (C18:0) acids were observed in FDB prepared with female chickens than male chickens ( $p<$ $0.05)$. FDB prepared with male birds had a significantly higher linoleic acid content whereas FDB prepared from females contained a greater level of arachidonic acid ( $p<$ 0.05). $\alpha$-Linolenic acid content was similar between the sexes. Linoleic (C18:2), $\alpha$-linolenic (C18:3), and arachidonic (C20:4) acid, as essential fatty acids, are considered very important for human health (Choe et al., 2010). Furthermore, arachidonic acid has flavor-enhancing proper-

Table 2. Nucleotide contents $(\mathrm{g} / \mathrm{kg})$ of freeze-dried broth made from male and female Korean native chickens

\begin{tabular}{ccccc}
\hline \hline Sex & Adenosine-5-monophosphate & Inosine-5-monophosphate & Inosine & Hypoxanthine \\
\hline Male & $2.60^{\mathrm{y}}$ & $12.40^{\mathrm{y}}$ & $15.20^{\mathrm{x}}$ & 4.70 \\
Female & $2.80^{\mathrm{x}}$ & $17.50^{\mathrm{x}}$ & $14.40^{\mathrm{y}}$ & 4.60 \\
SEM $^{1)}$ & 0.03 & 0.20 & 0.17 & 0.07 \\
\hline
\end{tabular}

${ }^{x, y}$ Means within a column with different superscripts differ significantly $(p<0.05)$.

${ }^{1)}$ Standard error of the mean $(n=12)$. 
Table 3. Fatty acid composition (\%) of freeze-dried broth made from male and female Korean native chickens

\begin{tabular}{cccc}
\hline \hline \multirow{2}{*}{ Fatty acid } & \multicolumn{2}{c}{ Sex } & \multirow{2}{*}{ SEM $^{1)}$} \\
\cline { 2 - 3 } & Male & Female & \\
\hline Myristic acid (C14:0) & $0.98^{\mathrm{x}}$ & $0.93^{\mathrm{y}}$ & 0.00 \\
Palmitic acid (C16:0) & $22.08^{\mathrm{y}}$ & $22.99^{\mathrm{x}}$ & 0.22 \\
Palmitoleic acid (C16:1) & $3.61^{\mathrm{x}}$ & $2.64^{\mathrm{y}}$ & 0.02 \\
Stearic acid (C18:0) & $6.44^{\mathrm{y}}$ & $7.88^{\mathrm{x}}$ & 0.19 \\
Oleic acid (C18:1) & 35.80 & 36.15 & 0.21 \\
Linoleic acid (C18:2) & $28.36^{\mathrm{x}}$ & $26.68^{\mathrm{y}}$ & 0.17 \\
Q-linolenic acid (C18:3) & 1.74 & 1.71 & 0.01 \\
Eicosenoic acid (C20:1) & 0.59 & 0.60 & 0.01 \\
Arachidonic acid (C20:4) & $0.30^{\mathrm{y}}$ & $0.32^{\mathrm{x}}$ & 0.00 \\
Docosahexaenoic acid & 0.08 & 0.09 & 0.01 \\
(C22:6) & $0.42^{\mathrm{y}}$ & $0.47^{\mathrm{x}}$ & 0.01 \\
SFA/USFA & $15.75^{\mathrm{x}}$ & $15.00^{\mathrm{y}}$ & 0.04 \\
n-6/n-3 &
\end{tabular}

$\overline{\mathrm{x}, \mathrm{y} \text { Means within a raw with different superscripts differ signifi- }}$ cantly $(p<0.05)$.

${ }^{1)}$ Standard error of the mean $(\mathrm{n}=12)$.

${ }^{2)}$ Saturated fatty acid/unsaturated fatty acid ratio.

Table 4. Free amino acid content $(\mathrm{g} / \mathrm{kg})$ of freeze-dried broth made from male and female Korean native chickens

\begin{tabular}{cccc}
\hline \hline \multirow{2}{*}{ Free amino acid } & \multicolumn{2}{c}{ Sex } & \multirow{2}{*}{ SEM $^{1)}$} \\
\cline { 2 - 3 } & Male & Female & \\
\hline Aspartic acid & 0.20 & 0.20 & 0.00 \\
Serine & $1.19^{\mathrm{x} 2)}$ & $1.09^{\mathrm{y}}$ & 0.02 \\
Glutamic acid & 0.60 & 0.64 & 0.03 \\
Glycine & $0.77^{\mathrm{x}}$ & $0.63^{\mathrm{y}}$ & 0.01 \\
Histidine & 0.46 & 0.44 & 0.01 \\
Arginine & 13.17 & 13.53 & 0.22 \\
Threonine & $9.57^{\mathrm{y}}$ & $11.00^{\mathrm{x}}$ & 0.20 \\
Alanine & $0.69^{\mathrm{x}}$ & $0.58^{\mathrm{y}}$ & 0.02 \\
Proline & 0.45 & 0.42 & 0.01 \\
Cysteine & 0.00 & 0.00 & 0.00 \\
Tryptophan & 0.64 & 0.64 & 0.01 \\
Valine & 0.33 & 0.34 & 0.01 \\
Methionine & 0.27 & 0.27 & 0.01 \\
Lysine & $0.29^{\mathrm{x}}$ & $0.21^{\mathrm{y}}$ & 0.02 \\
Isoleucine & 0.20 & 0.21 & 0.01 \\
Leucine & 0.43 & 0.45 & 0.01 \\
Phenylalanine & 0.55 & 0.54 & 0.01 \\
\hline
\end{tabular}

${ }_{\mathrm{x}, \mathrm{y}}$ Means within a raw with different superscripts differ significantly $(p<0.05)$.

${ }^{1)}$ Standard error of the mean $(\mathrm{n}=12)$.

ties (Jayasena et al., 2013b). Steamed minced meat and chicken broth prepared with Hinai-jidori chickens showed significantly higher scores for total flavor intensity and aftertaste when fed a diet supplemented with arachidonic acid (Kiyohara et al., 2011). In the present study, sex had no effect $(p>0.05)$ on the content of docosahexaenoic acid (C22:6; DHA), another flavor-related fatty acid (Jayasena et al., 2013a), in FDB. FDB prepared from females had a higher saturated fatty acid/unsaturated fatty acid ratio compared to that prepared from males $(p<0.05)$. However, n-6/n-3 ratio was significantly higher in FDB prepared with males.

\section{Free amino acid content}

The free amino acid content of FDB prepared with male and female $\mathrm{KNC}$ is presented in Table 4. Sixteen free amino acids were detected in the FDB samples, irrespective of the sex. However, only serine, glycine, threonine, alanine, and lysine levels differed significantly between sexes. No studies were found regarding the effect of sex on the free amino acid content of meat-based broth. Therefore, studies quantifying the same compounds in different meat essences and broth cubes were used to discuss the results of the present study. Arginine and threonine were the predominant free amino acids detected in both FDB samples in the current study. In contrast, Chiang et al. (2007) found that glutamic acid and serine were the predominant free amino acids in chicken broth cubes, whereas taurine and glutamic acids were prevalent in chicken essences (Wu and Shiau, 2002). Leucine, lysine, taurine, phenylalanine, and arginine were the predominant free amino acids in eel essence, whereas essences from hard clam and freshwater clam contained alanine as the major free amino acid (Wu and Shiau, 2002). Different raw materials and manufacturing methods used to produce FDB, meat essences, and broth cubes might result in variations in the free amino acid contents. FDB samples tested in this study had much higher levels of all free amino acids than did commercial chicken essences $(\mathrm{Wu}$ and Shiau, 2002) and chicken and pork broth cubes (Chiang et al., 2007). Beef essence, however, contained higher levels of aspartic acid, alanine, valine, glutamic acid, methionine, isoleucine, leucine, phenylalanine, and lysine (Wu and Shiau, 2002), compared to the FDB samples used in the present study.

Most free amino acids are associated with flavor development (Lim et al., 2013). Levels of sweet flavor-associated amino acids such as glycine, alanine, lysine, and serine (Lim et al., 2013) in the FDB samples were significantly different between sexes, with male chickens containing higher levels $(p<0.05)$ than female chickens (Table 4). However, the contents of free amino acids responsible for the umami flavor, glutamic and aspartic acids (Chiang et al., 2007), and those responsible for bitter taste such as valine, isoleucine, leucine, phenylalanine, methionine, arginine, and histidine (Lim et al., 2013), were comparable between FDB samples prepared with male and female 
$\mathrm{KNC}(p>0.05)$. In contrast, threonine levels in FDB from female birds were higher than in FDB made from male birds $(p<0.05)$. Jayasena et al. (2013b) reported that the meaty flavor of chicken broth is likely due to the presence of 2-methyl-3-furanthiol. The sulphur-containing amino acid, cysteine, is a major precursor for the formation of 2-methyl-3-furanthiol. Surprisingly, cysteine was absent in both FDB samples (Table 4), which may be attributed to the fact that the available cysteine might have been utilized completely for flavor development during preparation of the FDB.

\section{Conclusion}

Sex had no significant effect on creatine, betaine and carnitine levels in FDB prepared with KNC. Histidine dipeptides and cysteine were absent in both FDB samples. However, female chickens showed significantly higher levels of IMP and arachidonic acid in the broth samples than did the male chickens. FDB prepared with male $\mathrm{KNC}$ contained higher levels of inosine, linoleic acid, and sweet flavor-governing free amino acids such as glycine, alanine, lysine, and serine $(p<0.05)$. However, glutamic acid, oleic acid, and DHA were present in comparable amounts $(p>0.05)$ in FDB made from male and female KNC.

\section{Acknowledgements}

This work was supported by a grant from the NextGeneration BioGreen 21 Program (No. 00813302), Rural Development Administration, Republic of Korea.

\section{References}

1. Cambero, M. I., Pereira-Lima, C. I., Ordoñez, J. A., and García De Fernando, G. D. (2000) Beef broth flavour: Relation of components with the flavour developed at different cooking temperatures. J. Sci. Food Agric. 80, 1519-1528.

2. Chiang, P. D., Yen, C. T., and Mau, J. L. (2007) Non-volatile taste components of various broth cubes. Food Chem. 101, 932-937.

3. Choe, J. H., Nam, K. C., Jung, S., Kim, B., Yun, H. J., and Jo, C. (2010) Differences in the quality characteristics between commercial Korean native chickens and broilers. Korean $J$. Food Sci. An. 30, 13-19.

4. Constantin-Teodosiu, D., Howell, S., and Greenhaff, P. L. (1996) Carnitine metabolism in human muscle fiber types during submaximal dynamic exercise. J. Appl. Physiol. 80, 1061-1064.

5. De Zwart, F. J., Slow, S., Payne, R. J., Lever, M., George, P.
M., Gerrard, J. A., and Chambers, S. T. (2003) Glycine betaine and glycine betaine analogues in common foods. Food Chem. 83, 197-204.

6. Derave, W., Everaert, I., Beeckman, S., and Baguet, A. (2010) Muscle carnosine metabolism and beta-alanine supplementation in relation to exercise and training. Sports Med. 40, 247263.

7. Folch, J., Lees, M., and Stanley, G. H. S. (1957) A simple method for the isolation and purification of total lipides from animal tissues. J. Biol. Chem. 226, 497-509.

8. Gadekar, Y. P., Anjaneyulu, A. S. R., Thomas, R., Mendiratta, S. K., and Kondaiah, N. (2009) Quality changes in soup from deboned chicken frames at refrigerated $\left(4 \pm 1^{\circ} \mathrm{C}\right)$ and frozen $\left(18 \pm 1^{\circ} \mathrm{C}\right)$ storage. Int. J. Food Sci. Technol. 44, 1763-1769.

9. Guiotto, A., Calderan, A., Ruzza, P., and Borin, G. (2005) Carnosine and carnosine-related antioxidants: A review. Curr. Med. Chem. 12, 2293-2315.

10. Hughes, M. C., Kerry, J. P., Arendt, E. K., Kenneally, P. M., Mcsweeney, P. L. H., and O'neill, E. E. (2002) Characterization of proteolysis during the ripening of semi-dry fermented sausages. Meat Sci. 62, 205-216.

11. Jayasena, D. D., Ahn, D. U., Nam, K. C., and Jo, C. (2013a) Factors affecting cooked chicken meat flavour: A review. World Poultry Sci. J. 69, 515-526.

12. Jayasena, D. D., Ahn, D. U., Nam, K. C., and Jo, C. (2013b) Flavour chemistry of chicken meat: A review. Asian Austral. J. Anim. 26, 732-742.

13. Jayasena, D. D., Jung, S., Bae, Y. S., Kim, S. H., Lee, S. K., Lee, J. H., and Jo, C. (2014) Changes in endogenous bioactive compounds of Korean native chicken meat at different ages and during cooking. Poultry Sci. 93, 1842-1849.

14. Jung, S., Bae, Y. S., Kim, H. J., Jayasena, D. D., Lee, J. H., Park, H. B., Heo, K. N., and Jo, C. (2013) Carnosine, anserine, creatine, and inosine 5-monophosphate contents in breast and thigh meats from five lines of Korean native chicken. Poultry Sci. 92, 3275-3282.

15. Ke, L., Zhou, J., Lu, W., Gao, G., and Rao, P. (2011) The power of soups: Super-hero or team-work? Trends Food Sci. Technol. 22, 492-497.

16. Kiyohara, R., Yamaguchi, S., Rikimaru, K., and Takahashi, H. (2011) Supplemental arachidonic acid-enriched oil improves the taste of thigh meat of Hinai-jidori chickens. Poultry Sci. 90, 1817-1822.

17. Lim, D. G., Kim, K. T., Lee, K. H., Seo, K. S., and Nam, K. C. (2013) Physicochemical traits, fatty acid and free amino acid compositions of two-way crossbred pork belly. Korean J. Food Sci. An. 33, 189-197.

18. Mateescu, R. G., Garmyn, A. J., O’neil, M. A., Tait, R. G., Abuzaid, A., Mayes, M. S., Garrick, D. J., Van Eenennaam, A. L., Vanoverbeke, D. L., Hilton, G. G., Beitz, D. C., and Reecy, J. M. (2012) Genetic parameters for carnitine, creatine, creatinine, carnosine, and anserine concentration in longissimus muscle and their association with palatability traits in Angus cattle. J. Anim. Sci. 90, 4248-4255.

19. Mora, L., Hernández-Cázares, A. S., Sentandreu, M. A., and Toldrá, F. (2010) Creatine and creatinine evolution during 
the processing of dry-cured ham. Meat Sci. 84, 384-389.

20. Nam, K. C., Jo, C., and Lee, M. (2010) Meat products and consumption culture in the East. Meat Sci. 86, 95-102.

21. Purchas, R. W., Rutherfurd, S. M., Pearce, P. D., Vather, R., and Wilkinson, B. H. P. (2004) Concentrations in beef and lamb of taurine, carnosine, coenzyme $\mathrm{Q}_{10}$, and creatine. Meat Sci. 66, 629-637.

22. SAS (2012) SAS/STAT software for PC. Release 9.3, SAS Institute Inc., Cary, NC, USA.

23. Schmid, A. (2009) Bioactive substances in meat and meat products. Fleischwirtschaft 89, 83-90.

24. Sri Kantha, S., Takeuchi, M., Watabe, S., and Ochi, H. (2000) HPLC determination of carnosine in commercial canned soups and natural meat extracts. LWT-Food Sci. Technol. 33, 60-62.

25. Wei, W., Yongzhi, Z., Yu, S., and Muhan, Z. (2012) Compar- ative study on flavor quality of chicken soup made of different chicken breeds. Acta Agriculturae Jiangxi 24, 149-152.

26. Wu, H. C. and Shiau, C. Y. (2002) Proximate composition, free amino acids and peptides contents in commercial chicken and other meat essences. J. Food Drug Anal. 10, 170177.

27. Wyss, M. and Kaddurah-Daouk, R. (2000) Creatine and creatinine metabolism. Physiol. Rev. 80, 1107-1213.

28. Young, J. F., Therkildsen, M., Ekstrand, B., Che, B. N., Larsen, M. K., Oksbjerg, N., and Stagsted, J. (2013) Novel aspects of health promoting compounds in meat. Meat Sci. 95, 904-911.

29. Zeisel, S. H., Mar, M. H., Howe, J. C., and Holden, J. M. (2003) Concentrations of choline-containing compounds and betaine in common foods. J. Nutr. 133, 1302-1307.

(Received 2014.4.1/Revised 2014.6.12/Accepted 2014.6.17) 